\title{
High proportion of overt diabetes mellitus in pregnancy and missed opportunity for early detection of diabetes at a tertiary care centre in Pakistan
}

\author{
Aisha Syed Wali ${ }^{1}$, Raheela Rafique ${ }^{2}$, Sundus Iftikhar ${ }^{3}$, \\ Rakhshinda Ambreen ${ }^{4}$, Mohammad Yawar Yakoob ${ }^{5}$
}

\begin{abstract}
Objectives: To determine the frequency of diabetes in pregnancy (DIP), namely pre-gestational, gestational (GDM) and overt diabetes mellitus (DM) in women registered for delivery.

Methods: A retrospective chart review of antenatal women registered between January 01 to August 31, 2017 was performed. Gestational age, diagnosis of DIP, glucose levels at diagnosis and other relevant data was extracted. The effect of various fasting blood glucose (FBG) thresholds for diagnosis of DIP was assessed.

Results: DIP was diagnosed in $21.8 \%$ women (pre-gestational: $2 \%$, GDM: $81.2 \%$, overt DM: $16.8 \%$ ). In early registrants, $30.2 \%$ were detected through screening. However, $55.3 \%$ of women registered late. Women with pre-gestational DM were older, had more miscarriages, and greater personal and family history of diabetes versus GDM and overt DM. Raising the diagnostic threshold of FBG from $92 \mathrm{mg} / \mathrm{dl}$ to $95 \mathrm{mg} / \mathrm{dl}$ missed three women $(0.1 \%)$ and to $105 \mathrm{mg} / \mathrm{dl}$, missed six women $(0.2 \%)$.

Conclusion: We observed a high proportion of overt DM. In early registrants, almost one third of DIP was diagnosed in the first half of pregnancy, an opportunity missed in late registrants. Altering diagnostic thresholds of DIP affected only a small proportion of women.
\end{abstract}

KEYWORDS: Diabetes mellitus in pregnancy, Gestational diabetes, Overt diabetes in pregnancy, Pregestational diabetes.

doi: https://doi.org/10.12669/pjms.36.ICON-Suppl.1723

How to cite this:

Wali AS, Rafique R, Iftikhar S, Ambreen R, Yakoob MY. High proportion of overt diabetes mellitus in pregnancy and missed opportunity for early detection of diabetes at a tertiary care centre in Pakistan. Pak J Med Sci. Special Supplement ICON 2020. 2020;36(1):S38-S43. doi: https://doi.org/10.12669/pjms.36.ICON-Suppl.1723

This is an Open Access article distributed under the terms of the Creative Commons Attribution License (http://creativecommons.org/licenses/by/3.0), which permits unrestricted use, distribution, and reproduction in any medium, provided the original work is properly cited.

\footnotetext{
1. Aisha Syed Wali, FCPS.

Sheikh Saeed Memorial Hospital,

2. Raheela Rafique, MBBS.

Sheikh Saeed Memorial Hospital,

3. Sundus Iftikhar, MS.

4. Rakhshinda Ambreen, BDS.

5. Mohammad Yawar Yakoob, DS.

3-5: Indus Hospital Research Center,

1-5: Indus Health Network,

Karachi, Pakistan.

Correspondence:

Dr. Aisha Syed Wali, MBBS, FCPS,

The Indus Hospital,

Plot C-76, Sector 31/5,

Opposite Darussalam Society,

Korangi Crossing,

Karachi-75190, Pakistan.

E-mail: aisha.wali@tih.org.pk

* Received for Publication:

* Revision Received:

* Revision Accepted:
}

\section{INTRODUCTION}

Diabetes Mellitus (DM) is a major global health emergency of the 21st century. ${ }^{1}$ Diabetes in pregnancy (DIP) is also increasing along with other forms of diabetes mellitus. DIP can be classified into three types: (a) gestational diabetes mellitus (GDM), that develops during pregnancy; (b) overt DM, undiagnosed pre-existing diabetes mellitus that is detected in pregnancy; and (c) pregestational DM, seen in women with established diabetes who become pregnant. About $16.2 \%$ of live births are exposed to hyperglycemia in utero, $85.1 \%$ of which is due to GDM. ${ }^{2}$ The prevalence of GDM is highest in Southeast Asia, mostly in low or middle-income groups, estimated at approximately $24.2 \% .^{2}$ 
DIP not only adversely affects the fetus and the neonate, but also has long-term effects, including childhood obesity, metabolic syndrome and diabetes mellitus in adult life. ${ }^{1-3}$ This increases the disease burden. Good glycemic control is associated with better perinatal outcomes., ${ }^{4,5}$ The threshold of fasting plasma glucose (FPG) for the diagnosis of GDM has changed over the years and expecting better neonatal outcomes, the FPG criteria has lowered. ${ }^{1}$ Now in most of the institutions diagnosis of GDM is based on FPG of $92 \mathrm{mg} / \mathrm{dl}^{6-9}$ on recommendations of the "International Association of Diabetes and Pregnancy Study Groups" (IADPSG) 2010 criteria. ${ }^{10}$

At The Indus Hospital (TIH) Karachi, the IADPSG 2010 criteria $^{10}$ is followed for screening and diagnosis of diabetes in pregnancy. As such, it is based on FBG, glycated hemoglobin (HbA1c) or a 2-hour $75 \mathrm{~g}$ oral glucose tolerance test (OGTT) according to gestational age and risk factors. We conducted this study to determine the burden and frequency of GDM, overt DM and pre-gestational $\mathrm{DM}$ amongst population that we register at $\mathrm{TIH}$. We also compared the difference between early and late screened patients. Finally, we compared the frequency of GDM at different thresholds of FPG i.e. $105 \mathrm{mg} / \mathrm{dl}, 95 \mathrm{mg} / \mathrm{dl}$ and $92 \mathrm{mg} / \mathrm{dl}$.

\section{METHODS}

A retrospective observational study was conducted at TIH, a tertiary care centre located in Korangi, Karachi, Pakistan. This hospital provides free-of-cost quality care to underprivileged patients. Electronic medical records of women registered for delivery from January 1, 2017 to August 31, 2017 and who were less than 34 weeks by the end of study period, were retrieved from Hospital Management Information System (HMIS). Data was collected manually on forms designed for the study. It included demographic data, DM screening results, gestational age at booking and screening. All necessary ethical approvals were in place (IRD_ IRB_2017_10_003).

Women with known diagnoses with type 1 or type 2 diabetes at the time of registration were labeled "pre-gestational DM". All other registered pregnant women were screened. At the first prenatal visit at less than 24 weeks gestation, FPG was used as early screening and diagnostic tool of DIP. Glycated hemoglobin (HbA1c) was additionally checked in high-risk groups (i.e. diabetes in first degree relative, previous history of GDM, body mass index $>30 \mathrm{~kg} / \mathrm{m}^{2}$, known polycystic ovarian syndrome (PCOS), previous history of macrosomia, unexplained stillbirth or neonatal death). ${ }^{10}$ As per protocol, women with PCOS on metformin, stopped this drug two weeks prior to taking the screening test. In accordance with IADPSG criteria, ${ }^{10}$ women were categorized into three groups. Those with FPG $<92 \mathrm{mg} / \mathrm{dl}$ were labeled non-diabetic; those with FPG $92 \mathrm{mg} / \mathrm{dl}-$ $125 \mathrm{mg} / \mathrm{dl}$ as GDM and those with FPG $\geq 126 \mathrm{mg} /$ $\mathrm{dl}$ and/or an HbA1c of $\geq 6.5 \%$ as overt DM. Women presenting for registration at 24 weeks' gestation or later along with those who tested negative at early screening were screened at 24-28 weeks' gestation with a 2 hour 75-g OGTT. Using OGTT, any one or more of the following criteria met at any time in pregnancy is diagnostic of DIP; FPG $\geq 92 \mathrm{mg} /$ $\mathrm{dl}$, 1-hour glucose $\geq 180 \mathrm{mg} / \mathrm{dl}$, 2-hour glucose $\geq$ $153 \mathrm{mg} / \mathrm{dl}^{10}{ }^{10}$ Women not tolerating OGTT were screened with $50 \mathrm{~g}$ Oral Glucose Challenge Test (GCT); with a threshold for GDM being 1-hour glucose level $\geq 140 \mathrm{mg} / \mathrm{dl}^{11}$

All pregnant women with diagnosed pregestational type 1 and type 2 diabetes mellitus at the time of registration and those diagnosed as GDM or overt DM on the basis of the abovementioned screening were included in the sample. Women with secondary diabetes mellitus and those on steroid therapy were excluded. Outcomes measures were frequency of DIP, including frequency of pre-gestational DM, GDM and overt DM. The detection of GDM and overt DM on early and late screening was analyzed. The frequency of GDM detected using FPG $\geq 92 \mathrm{mg} / \mathrm{dl}$ was also compared with frequency on of GDM using FPG $\geq 95 \mathrm{mg} / \mathrm{dl}$ and FPG $\geq 105 \mathrm{mg} / \mathrm{dl}$ as diagnostic thresholds.

Data was entered and analysed using SPSS version 21.0. A descriptive analysis was conducted for quantitative variables after assessment for normality. Normally distributed variables were reported as mean \pm standard deviation and nonnormal variables were reported as median (with Inter Quartile Range, IQR). The frequency and percentage of qualitative variables was assessed. Student t-test was used to compare continuous normal variables, Mann-Whitney U-test for continuous non-normal variables and Chi-square or Fisher's exact test was used for categorical variables. All p-values were two-sided and considered statistically significant if less than 0.05 . 


\section{RESULTS}

A total of 2462 women were registered during the study period of eight months. A diagnosis of DIP was made in $537(21.8 \%)$. The frequency of pre-gestational DM was $0.5 \%(n=11 / 2462)$; among whom, one woman had Type-1 and ten had Type$2 \mathrm{DM}$. The remaining 2451 were screened for DM and $526(21.5 \%)$ were positive. Of these, $17.8 \%$ $(436 / 2451)$ had GDM and 3.7\% $(90 / 2451)$ had overt DM. Of all women with DIP $(n=537)$, overt DM was seen in $16.8 \%(n=90)$, GDM in $81.2 \%(n=436)$ and remaining $2 \%(\mathrm{n}=11)$ were pre-gestational.

Of those who were screened positive for DIP, $44.7 \%$ women $(n=235)$ were early bookers, i.e. registered within 24 weeks gestation. Of these, $30.2 \%(n=71 / 235)$ were diagnosed with DIP on initial screen, while the remaining $69.8 \% \quad(n=164)$ were screened negative at early screening for DIP but found to be positive on later screen at 24-28 weeks gestation (Fig.1).

The distribution of demographics and risk factors across categories of DIP is shown in Table-I. Women with pre-existing DM were older compared to women with GDM and overt DM. They had a history of two or more miscarriages, GDM in the past and a family history of diabetes compared to those with GDM and overt DM ( $\mathrm{p}<0.05)$.

Applying the threshold of FPG to $95 \mathrm{mg} /$ $\mathrm{dl}$, three cases of GDM $(0.1 \%)$ would have been missed while applying a threshold of $105 \mathrm{mg} / \mathrm{dl}$, 6 cases of GDM $(0.2 \%)$ would have been missed in 2451 women screened (Table-II).

\section{DISCUSSION}

Our study set out to determine the burden of diabetes in the pregnant population we serve. Our overall finding of $21.8 \%$ of registered pregnant women suffering from DIP correlates with prevalence in Southeast Asian population estimated at $24.2 \% .^{2}$ The proportion of GDM among the women suffering from DIP was $81.2 \%$ and is consistent with the global proportion of GDM cited as $86.4 \%$ of total glucose intolerance in pregnancy. ${ }^{2}$ It must be emphasized that $16.8 \%$ of women had overt diabetes mellitus in our screening program - a large proportion of young women unaware of their disease. Comparatively, $7-8 \%$ of women worldwide have overt DM when screened in this way. ${ }^{2}$ Our results may reflect a lack of health awareness or education in our patient population. There may be poor access to primary health care in low-income settings as seen in the communities served by TIH. The high prevalence of DIP strongly warrants advocacy for primary and secondary prevention of diabetes, as recommended by the International Diabetes Federation. $^{2}$

The frequency of GDM $(17.7 \%)$ in our study is comparable to national data from Pakistan including Bahawalpur in $2012(19.0 \%)^{12}$ and the Karachi/Hyderabad region in 2013-2016 (11.8\%). ${ }^{13}$ Southeast Asian countries such as Vietnam $(20.1 \%){ }^{14}$ Singapore $(18.9 \%),{ }^{14}$ China $(11.9 \%)^{14}$ and Malaysia $(11.8 \%)^{14}$ also report similar figures. However, other regional neighbors have reported variable results, depending on the diagnostic criteria used. A study from India ${ }^{15}$ reported prevalence to be as low as $7.1 \%$ based on American Diabetes Association criteria. Similarly, in Nepal, ${ }^{16}$ WHO and IADPSG criteria for diagnosis was compared and prevalence of $2.5 \%$ and $6.6 \%$ respectively were found. However, a study from

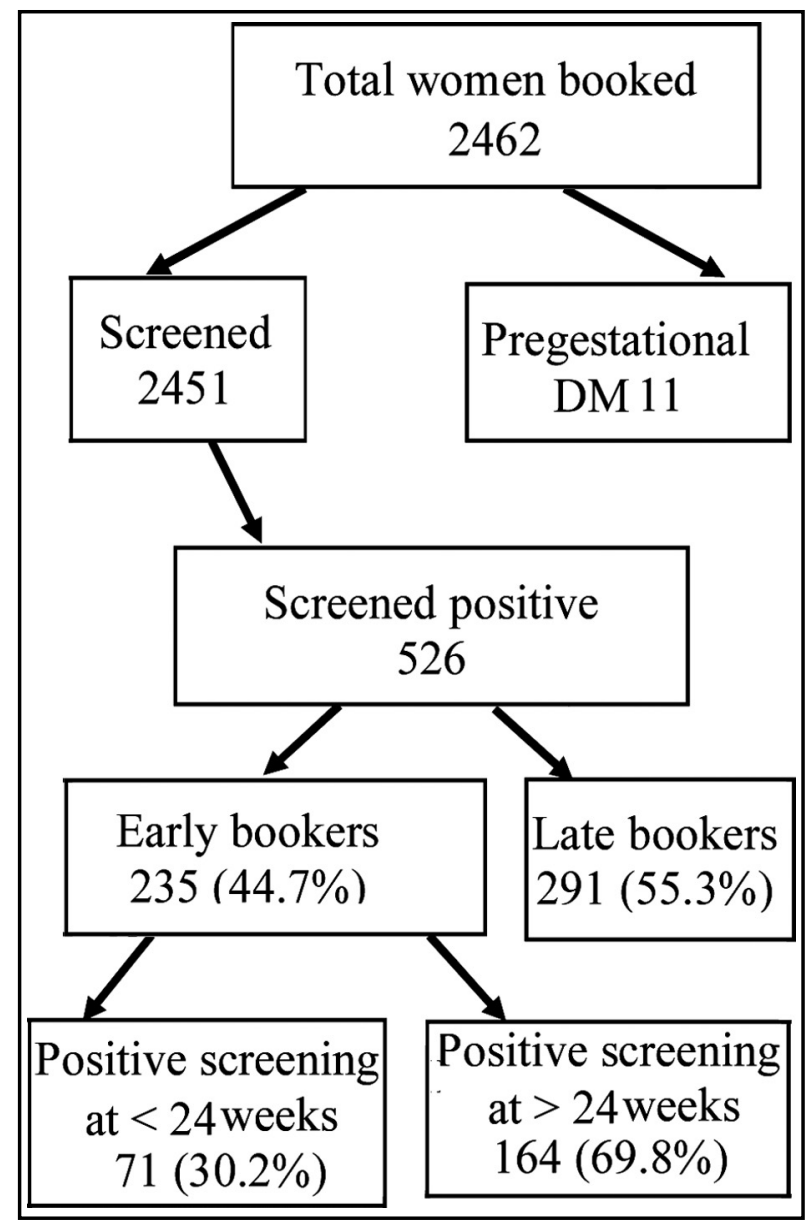

Fig.1: Flow-diagram showing the diagnosis of diabetes in pregnancy with early vs. late booking. 
Aisha Syed Wali et al.

Table-I: The distribution of demographics and risk factors across categories of DIP.

\begin{tabular}{|c|c|c|c|c|}
\hline & $\begin{array}{l}\text { Pre-gestational } \\
\operatorname{DM} n(\%)\end{array}$ & $\begin{array}{l}\text { GDM } \\
n(\%)\end{array}$ & $\begin{array}{l}\text { Overt DM } \\
n(\%)\end{array}$ & P-value \\
\hline Age in years (median) & $32(30-35)$ & $26(23-30)$ & $28(25-31.5)$ & $<0.001^{* *}$ \\
\hline $\begin{array}{l}\text { Gestational age in weeks median (IQR) } \\
\text { At booking } \\
\text { At diagnosis }\end{array}$ & $23(12-26)$ & $\begin{array}{l}24(20-27) \\
26(24-28)\end{array}$ & $\begin{array}{c}24.5(21-26) \\
25.5(24-27.25)\end{array}$ & $\begin{array}{l}0.465 \\
0.185\end{array}$ \\
\hline $\begin{array}{l}\text { Education level } \\
\text { Illiterate } \\
\text { Below matric } \\
\text { Matric } \\
\text { Intermediate } \\
\text { Graduate } \\
\text { Post-graduate }\end{array}$ & $\begin{array}{c}0(0) \\
4(50.0) \\
3(37.5) \\
2(25) \\
2(25) \\
0(0)\end{array}$ & $\begin{array}{c}48(11.4) \\
126(29.8) \\
144(34.1) \\
68(16.1) \\
30(7.1) \\
6(1.4)\end{array}$ & $\begin{array}{c}11(12.2) \\
155(29.8) \\
31(34.4) \\
12(13.3) \\
8(8.9) \\
0(0)\end{array}$ & 0.834 \\
\hline $\begin{array}{l}\text { Substance use } \\
\text { None } \\
\text { Cigarette smoking } \\
\text { Betel nuts } \\
\text { Chewable tobacco }\end{array}$ & $\begin{array}{c}11(100) \\
0(0) \\
0(0) \\
0(0)\end{array}$ & $\begin{array}{c}377(88.1) \\
1(0.2) \\
48(11.2) \\
2(0.5)\end{array}$ & $\begin{array}{c}81(94.2) \\
0(0) \\
5(5.8) \\
0(0)\end{array}$ & 0.249 \\
\hline $\begin{array}{l}\text { Parity } \\
\text { Nullipara } \\
\text { Primigravida } \\
\text { Multigravida }\end{array}$ & $\begin{array}{l}1(9.1) \\
3(27.3) \\
7(63.6)\end{array}$ & $\begin{array}{l}154(35.4) \\
130(29.9) \\
151(34.7)\end{array}$ & $\begin{array}{l}23(25.6) \\
30(33.3) \\
37(41.1)\end{array}$ & 0.107 \\
\hline $\begin{array}{l}\text { Past history of miscarriages } \\
\text { Overall miscarriage } \\
\geq 2 \text { miscarriages }\end{array}$ & $\begin{array}{l}4(36.4) \\
3(27.3)\end{array}$ & $\begin{array}{c}105(24.1) \\
21(4.8)\end{array}$ & $\begin{aligned} 27(30) \\
6(6.7)\end{aligned}$ & $\begin{array}{c}0.371 \\
<0.01^{* *}\end{array}$ \\
\hline Past history of premature delivery (<37 weeks) & $1(9.1)$ & $3(0.7)$ & $1(1.1)$ & 0.088 \\
\hline Past history of low birth weight $(<2.5 \mathrm{~kg})$ & $0(0)$ & $0(0)$ & $1(1.1)$ & 0.188 \\
\hline Past history of birth weight $>3.5 \mathrm{~kg}$ & $0(0)$ & $2(0.5)$ & $0(0)$ & 1.00 \\
\hline Past history of congenital anomaly & $0(0)$ & $3(0.7)$ & $0(0)$ & 1.00 \\
\hline Past history of intra-uterine/neonatal death & $0(0)$ & $28(6.4)$ & $5(5.6)$ & 0.66 \\
\hline Past history of GDM & $3(27.3)$ & $7(1.6)$ & $1(1.1)$ & $0.001^{* *}$ \\
\hline $\begin{array}{l}\text { Past history of pregnancy-induced } \\
\text { hypertension }\end{array}$ & $0(0)$ & $14(3.2)$ & $3(3.3)$ & 1.00 \\
\hline Past history of pre-eclampsia & $0(0)$ & $8(1.8)$ & $1(1.1)$ & 1.00 \\
\hline Past history of C-section & $4(36.4)$ & $94(21.6)$ & $25(27.8)$ & 0.248 \\
\hline Family history of diabetes & $6(54.5)$ & $91(21.1)$ & $32(35.6)$ & $0.001^{* *}$ \\
\hline Family history of hypertension & $2(18.2)$ & $86(20)$ & $11(12.5)$ & 0.263 \\
\hline Family history of pre-eclampsia & $0(0)$ & $1(0.2)$ & $1(1.1)$ & 0.342 \\
\hline
\end{tabular}

** $\mathrm{P}$ value $<0.05$.

Bangladesh $^{17}$ reported GDM as $36 \%$ according to WHO-1999 criteria.

We observed that $55.3 \%$ of women diagnosed with DIP registered after 24 weeks of gestation. In women who registered earlier than 24 weeks of gestation, $30.2 \%$ of women were diagnosed with DIP on early screening. A large proportion of late registrants may have missed this opportunity for detection and control DIP earlier in pregnancy. Larger prospective studies are required to infer the proportion of DIP detected in early pregnancy, however early detection is important to prevent in utero exposure to hyperglycemia. This further emphasizes the need for advocacy for early 
Table-II: Diagnosis of DIP at variable diagnostic criteria of fasting plasma glucose (FPG) in mg/dl.

\begin{tabular}{lccc}
\hline & $\begin{array}{c}\text { Cut-off } \\
F P G \geq 92 \\
m g / d l\end{array}$ & $\begin{array}{c}\text { Cut-off } \\
F P G \geq 95 \\
m g / d l\end{array}$ & $\begin{array}{c}\text { Cut-off } \\
F P G \geq 105 \\
m g / d l\end{array}$ \\
\hline $\begin{array}{c}\text { Number of } \\
\text { non-diabetic women } \\
\begin{array}{c}\text { Number of } \\
\text { women with GDM }\end{array}\end{array}$ & 0 & 3 & 6 \\
\hline
\end{tabular}

registration and screening for diabetes in our population to enable secondary prevention of DIP. The high frequency of DIP and its rising trend highlights the need for primary prevention at the community level as well, promoting lifestyle modifications and healthy eating habits in young girls.

Over recent decades, there has been tightening of the diagnostic criteria for GDM in an attempt to improve neonatal outcomes. World Health Organization considered FPG $\geq 140 \mathrm{mg} / \mathrm{dl}$ in 1999, FPG $\geq 126 \mathrm{mg} / \mathrm{dl}$ in 2006, ${ }^{18}$ NICE guideline recommended FPG $\geq 105 \mathrm{mg} / \mathrm{dl}$ in $2008,{ }^{19}$ and the IADPSG $^{20}$ consensus was set at FPG $\geq 95 \mathrm{mg} /$ $\mathrm{dl}$ as the diagnostic criteria for GDM but further lowered to diagnostic cut off to $\geq 92 \mathrm{mg} / \mathrm{dl}$ in $2010^{10}$ and now most institutions, including those mentioned above and ours, have adopted this. However studies are emerging that question the benefit of lowering the FPG with respect to fetal outcomes as costs of over-diagnosis and over-medication can burden health systems, in addition to increasing anxiety. ${ }^{21-25}$ Comparing our frequency of GDM based on IADPSG 2010 criteria with those of different thresholds of FPG, we found relatively small numbers of women being affected. There is a need for larger studies to define the diagnostic criteria for GDM that best correlates with good neonatal outcomes in the South-East Asian population while balancing cost and burden on healthcare.

\section{CONCLUSION}

We observed a high frequency of DIP with a higher proportion of overt DM. About one third of cases of DIP were diagnosed in the first half of pregnancy, an opportunity that was missed in women who registered late. The effect of varying diagnostic criteria on frequency of GDM was small and warrants further studies to assess its significance.
Acknowledgements: The authors would like to acknowledge the managements of IHRC and Sheikh Saeed Memorial Hospital of Indus Hospital Network for their services and support.

\section{Sources of Funding: None.}

Conflicts of Interest: None

\section{REFERENCES}

1. Group IDFDA. Update of mortality attributable to diabetes for the IDF Diabetes Atlas: Estimates for the year 2013. Diabetes Res Clin Pract. 2015;109(3):461-465. doi: 10.1016/j.diabres.2015.05.037

2. Federation ID. IDF Diabetes Federation. 2017.

3. Iqbal R, Jawaid S, Jabbar A, Qureshi R. Gestational diabetes mellitus--a forerunner of chronic disorders in mother and child. J Pak Med Assoc. 2009;59(7):478-482.

4. Horvath K, Koch K, Jeitler K, Matyas E, Bender R, Bastian $\mathrm{H}$, et al. Effects of treatment in women with gestational diabetes mellitus: systematic review and meta-analysis. BMJ. 2010;340(11):c1395. doi: 10.1136/ bmj.c1395

5. Gandhi P, Bustani R, Madhuvrata $\mathrm{P}$, Farrell $\mathrm{T}$. Introduction of metformin for gestational diabetes mellitus in clinical practice: Has it had an impact? Euro J Obstet Gynecol Reprod Biol. 2012;160(2):147-150. doi: 10.1016/j.ejogrb.2011.11.018

6. Morley L, Simpson N. The principles of screening tests as applied to obstetrics and gynaecology. J Reprod Med. 2016;26(1):1-6. doi: 10.1016/j.ogrm.2015.11.005

7. Dberkus M, Langer O, Mpiper J, Luther M. Efficiency of lower threshold criteria for the diagnosis of gestational diabetes. Obstet Gynecol. 1995;86(6):892-896. doi: 10.1016/0029-7844(95)00319-m

8. d'Emden M. Do the new threshold levels for the diagnosis of gestational diabetes mellitus correctly identify women at risk? Diabetes Care. 2014;37(2):e30. doi: $10.2337 / \mathrm{dc} 13-2234$

9. Blumer I, Hadar E, Hadden DR, Jovanovic L, Mestman $\mathrm{JH}$, Murad $\mathrm{MH}$, et al. Diabetes and pregnancy: an endocrine society clinical practice guideline. J Clin Endocrinol Metab. 2013;98(11):4227-4249. doi: 10.1210/ jc. 2013-2465

10. International Association of $\mathrm{D}$, Pregnancy Study Groups Consensus P, Metzger BE, Gabbe SG, Persson $\mathrm{B}$, Buchanan TA, et al. International association of diabetes and pregnancy study groups recommendations on the diagnosis and classification of hyperglycemia in pregnancy. Diabetes Care. 2010;33(3):676-682. doi: $10.2337 / \mathrm{dc} 09-1848$

11. AmericanDiabetesA.Diagnosisandclassification of diabetes mellitus. Diabetes Care. 2010;33(Supplement_1):S62-S69. doi: 10.2337/dc10-S062

12. Zaman N, Taj N, Nazir S, Ullah E, Fatima N. Gestational Diabetes Mellitus and Obesity: An experience at a teaching hospital in Bahawalpur, Pakistan. Pak J Med Res. 2013;38(2):165-168.

13. Riaz M, Nawaz A, Masood SN, Fawwad A, Basit A, Shera AS. Frequency of gestational diabetes mellitus using DIPSI criteria, a study from Pakistan. CEGH. 2019;7(2):218-221. doi: 10.1016/j.cegh.2018.06.003 
14. Nguyen CL, Pham NM, Binns CW, Duong DV, Lee AH. Prevalence of Gestational Diabetes Mellitus in Eastern and Southeastern Asia: A Systematic Review and Meta-Analysis. J Diabetes Res. 2018;2018:6536974. doi: $10.1155 / 2018 / 6536974$

15. Rajput R, Yadav Y, Nanda S, Rajput M. Prevalence of gestational diabetes mellitus \& associated risk factors at a tertiary care hospital in Haryana. Indian J Med Res. 2013;137(4):728-733.

16. Thapa $P$, Shrestha S, Flora M, Bhattarai M, Thapa N, Mahat B, et al. Gestational Diabetes Mellitus "A Public Health Concern in Rural Communities of Nepal. J Nepal Health Res Counc. 2016. Vol No paged number missing (incomplete page no)

17. N. S, editor Prevalence of gestational diabetes mellitus and its risk factors: Bangladesh perspective. Biomed Res. Joint Event on 2nd World Obesity Congress \& International Conference on Diabetes and Endocrinology \& 2nd World Vaccines and Immunology Congress. 2018; Tokyo, Japan

18. Organization WH. Definition, diagnosis and classification of diabetes mellitus and its complications: report of a WHO consultation. Part 1, Diagnosis and classification of diabetes mellitus. Geneva: World Health Organization; 1999.

19. Walker JD. NICE guidance on diabetes in pregnancy: management of diabetes and its complications from preconception to the postnatal period. NICE clinical guideline 63. London, March 2008. Diabet Med. 2008;25(9):1025-1027. doi: 10.1111/j.14645491.2008.02532.x

20. Metzger BE, Buchanan TA, Coustan DR, de Leiva A, Dunger DB, Hadden DR, et al. Summary and recommendations of the Fifth International WorkshopConference on Gestational Diabetes Mellitus. Diabetes Care. 2007;30(Suppl-2):S251-260. doi: 10.2337/ dc07-s225
21. Rani PR, Begum JJJoc. Screening and diagnosis of gestational diabetes mellitus, where do we stand. JCDR. 2016;10(4):QE01

22. Moses RG, Morris GJ, Petocz P, San Gil F, Garg D. The impact of potential new diagnostic criteria on the prevalence of gestational diabetes mellitus in Australia. Med J Aust. 2011;194(7):338-340.

23. Ehmann DMT, Hickman PE, Potter JM. Are the changes in diagnostic criteria for gestational diabetes mellitus reflected in perinatal outcomes? A retrospective assessment. Aust N Z J Obstet Gynaecol. 2019. doi: 10.1111/ajo.12956 (incomplete page no)

24. Behboudi-Gandevani S, Amiri M, Bidhendi Yarandi R, Ramezani Tehrani F. The impact of diagnostic criteria for gestational diabetes on its prevalence: a systematic review and meta-analysis. Diabetol Metab Syndr. 2019;11(1):11. doi: 10.1186/s13098-019-0406-1

25. Meloncelli NJL, Barnett AG, De Jersey SJ. 1437-P: How Has Changing the Diagnostic Criteria Impacted Perinatal Outcomes for Those NOT Diagnosed with GDM? Diabetes. 2019;68(Suppl 1):1437-P. doi: 10.2337/db19-1437-P

\section{Authors' Contributions:}

ASW: Developed the research protocol and most of the manuscript writing, takes responsibility for integrity of research.

MYY: Did literature search, references and helped in writing and editing manuscript.

RR \& RA: Assisted in writing introduction, methods and results.

SI: Analysed the data and wrote the analysis. 